\title{
Database Lock and Unlock Approval
}

National Cancer Institute

\section{Source}

National Cancer Institute. Database Lock and Unlock Approval. NCI Thesaurus. Code C115626.

Records that confirm the completion and satisfaction of all the requirements for a database release. 